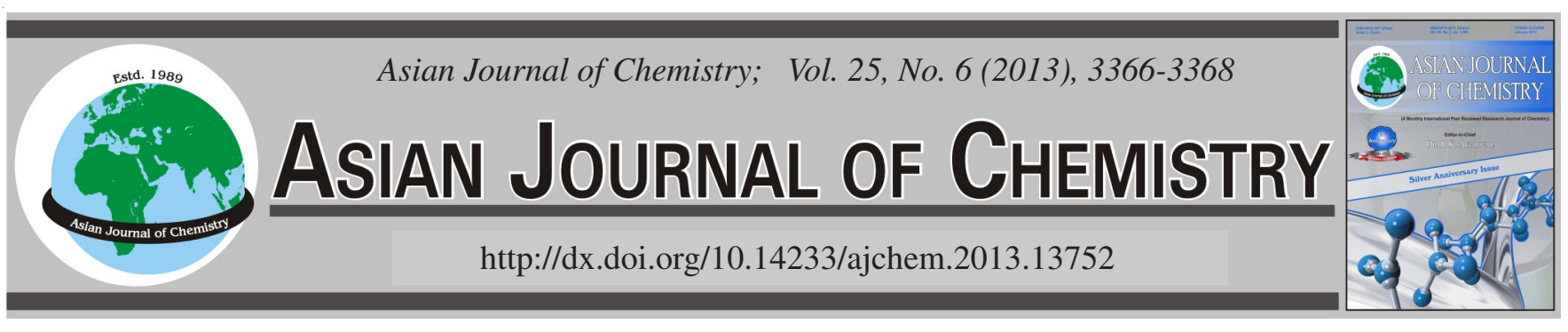

\title{
Preparation and Magnetic Properties of the Flake-Like Nickel Nanocrystals
}

\author{
Q. CHEN* and D.H. YAO
}

Chemical Engineering and Pharmaceutics School, Henan University of Science and Technology, Luoyang, P.R. China

*Corresponding author: Fax: +86 379 64231368; Tel: +86 379 64231914; E-mail: chenquan05@126.com

Flake-like nickel nanocrystals with a diameter of approximately $1 \mu \mathrm{m}$ were successfully obtained by reducing nickel salts in the presence of hydrazine hydrate under normal pressure. The flake-like nickel was characterized utilizing X-ray diffraction and scanning electron microscopy. Moreover, it was revealed that the novel flake-like nickel nanocrystals were of ferromagnetism with the saturation magnetization of $21.3 \mathrm{emu} / \mathrm{g}$ and the coercivity of 204.3 Oe by the magnetic hysteresis measurement at room temperature. The present approach has the advantages of fast reaction rate and low cost. The resulting nickel flakes might be potential as catalysts, magnetic storage materials and conductive fillers for shielding of electromagnetic interference.

Key Words: Nickel, Nanostructure, Flakes, Magnetic materials.

\section{INTRODUCTION}

In recent years, research on nanosized metal magnetic materials has been highlighted extensively owing to the anticipated intriguing properties of them compared to the bulk stuffs ${ }^{1-4}$. Particularly, anisotropic magnetic nanoscale materials have attracted worldwide attention because they can be potentially applied in preparing magnetic sensors, memory devices, catalysts and galvanomagnetic materials ${ }^{5-8}$. Metallic nickel possesses inherent magnetism as a permanent magnetic material and the synthesis of nanosized nickel with various shapes has been realized by different approaches ${ }^{9,10}$. Besides, morphology control of nickel has also been actively investigated, in which hollow microspheres, triagonal nanoparticles, flower shaped nanocrystals as well as other $1 \mathrm{D}$ nickel nanostructures including single-crystal nanobelts and polycrystalline wires have been successfully synthesized by different ways ${ }^{11-15}$.

Fabricating the metal flakes is mainly dependent on the mechanical grinding and template techniques. However, the flakes obtained by the former method often suffer from the accompanied generation of impurities and the nonuniform morphologies and sizes. Meanwhile, the latter method is also unfavourable resulted from the complex synthetic procedures concerning the purification (removing the templates) of the final products. Thus, it is of great need to find out a simple and convenient method to fabricate the flake-like magnetic nanomaterials to both simplify the overall synthesis and avoid introducing impurities. In the present work, flake-like $\mathrm{Ni}$ nanocrystallites were prepared by simply reducing nickel chloride with hydrazine hydrate under normal pressure to verify the above assumption. The flake-like Ni was characterized by SEM and XRD and its magnetic properties were also measured. The relevant research being dealt with herein provide a novel common method for preparing a variety of other well-defined magnetic metal nanostructures with controllable morphologies, which will further exploit the unique properties and peculiar applications comprehensively.

\section{EXPERIMENTAL}

All the analytical pure reagents were directly utilized without further purification. Typically, $0.12 \mathrm{~g}$ of nickel chloride $\left(\mathrm{NiCl}_{2} \cdot 6 \mathrm{H}_{2} \mathrm{O}\right.$, Tianjin Kermel Chemical Co. Ltd., China) and $0.11 \mathrm{~g}$ sodium dodecyl benzene sulfonate (SDBS, Tianjin Kermel Chemical Co. Ltd., China) were first dissolved in $50 \mathrm{~mL}$ of ethylene glycol (EG, Tianjin Kermel Chemical Co. Ltd., China) to yield a green transparent solution, allowing $\mathrm{Ni}^{2+}$ to disperse homogeneously. $1 \mathrm{~mL}$ of hydrazine hydrate solution $\left(\mathrm{N}_{2} \mathrm{H}_{4} \cdot \mathrm{H}_{2} \mathrm{O}\right.$, concentration 80 wt \%, Tianjin Kermel Chemical Co. Ltd., China) was then added drop wise into the reseda solution while being stirred continuously. A lavender solution was gradually generated within several minutes, to which $0.10 \mathrm{~g} \mathrm{NaOH}$ (Tianjin Deen Chemical Co. Ltd., China) was then added drop wise to produce a sky-blue solution in several minutes. The resulting solution was then heated to $60{ }^{\circ} \mathrm{C}$ and maintained for several minutes under vigorous stirring. A few minutes later, a clear and colourless solution was obtained and the desired product was depositing as black solids at the 
same time, indicating that metallic Ni was forming. The chemical reaction for the synthetic procedure above is expressed below:

$$
2 \mathrm{Ni}^{2+}+\mathrm{N}_{2} \mathrm{H}_{4}+4 \mathrm{OH}^{-}=2 \mathrm{Ni}+\mathrm{N}_{2} \uparrow+4 \mathrm{H}_{2} \mathrm{O}
$$

After the reaction, the products were collected by magnetic separation and washed several times with alcohol to eliminate the possibly remained ions and other materials. Then the products were finally dried under vacuum at $60{ }^{\circ} \mathrm{C}$ for $12 \mathrm{~h}$, which were characterized utilizing a Philips X' Pert Pro X-ray diffractometer (XRD, CuK $\alpha$ radiation, $\lambda=0.15418 \mathrm{~nm}$ ) and a JEOL JSM-5600LV scanning electron microscope (SEM, acceleration voltage $20 \mathrm{kV}$ ). The magnetic hysteresis loop of the product at room temperature was measured by a samplevibrating magnetometer (VSM, Lake Shore 7400, USA).

\section{RESULTS AND DISCUSSION}

XRD patterns of the typical Ni samples prepared are shown in Fig. 1. Three characteristic peaks of $\mathrm{Ni}\left(2 \theta=44.5^{\circ}\right.$, $51.8^{\circ}$ and $76.4^{\circ}$ ) discerned for both samples corresponded to Miller indices (111), (200) and (220), respectively, suggesting that they both consisted of pure face-centered cubic (fcc) Ni (PDF standard cards, JCPDS 01-1260, space group Fm³ without impurities. Although nickel is readily oxidized, oxide peaks were not observed, which might be attributed to the appropriate $\mathrm{pH}$, temperature and hydrazine concentration utilized in the reaction as well as the generation of inactive $\mathrm{N}_{2}$ as the protective atmosphere.

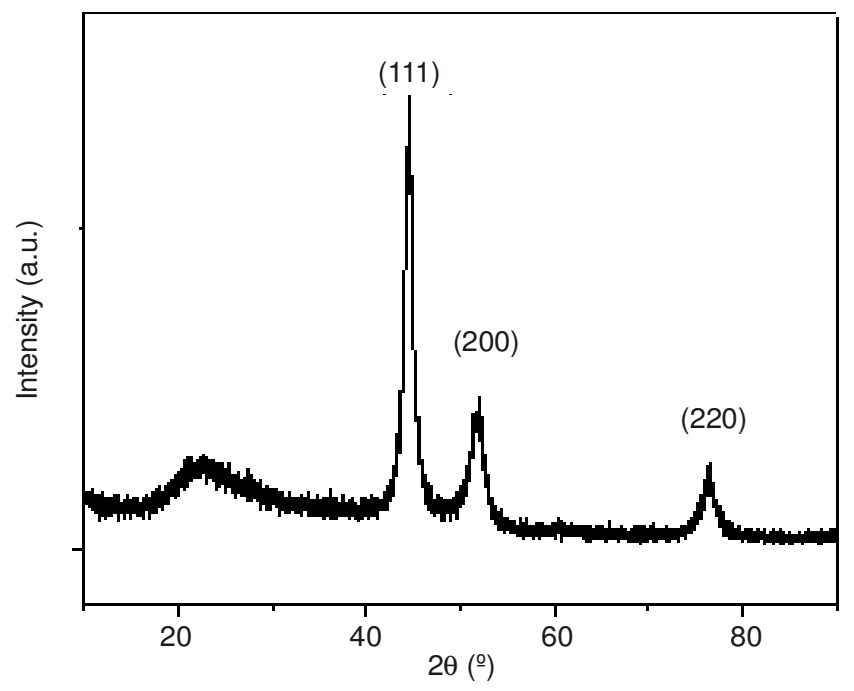

Fig. 1. XRD patterns of the final Ni samples obtained at the end of the reaction

SEM morphologies of the Ni samples are exhibited in Fig. 2, in which Fig. 2A shows that all the Ni samples adopt a flake-like configuration. Fig. 2B showing the magnified SEM image denotes that all the Ni flakes are composed by about 1 $\mu \mathrm{m}$ disc-like closely contacted particles. Taking into consideration that the formation of Ni flakes significantly depend on the intrinsic growth behaviours of $\mathrm{Ni}$ crystals and the utilized experimental conditions such as concentrations of the reactants, temperatures, types of surfactants, etc. Therefore, the growing mechanism of the Ni flakes is sophisticated and has not been well clarified yet. Fu et al. have synthesized chain- like CoNi alloy assemblies hydrothermally assisted by a surfactant and speculated that the surfactants cetyltrimethylamonium bromide (CTAB) or cetyltrimethyl-ammonium chloride (CTAC) were able to act as the template to yield the chain-like structure $^{16}$. Similarly, generation of the flake-like products might be issued from the SDBS micelle.

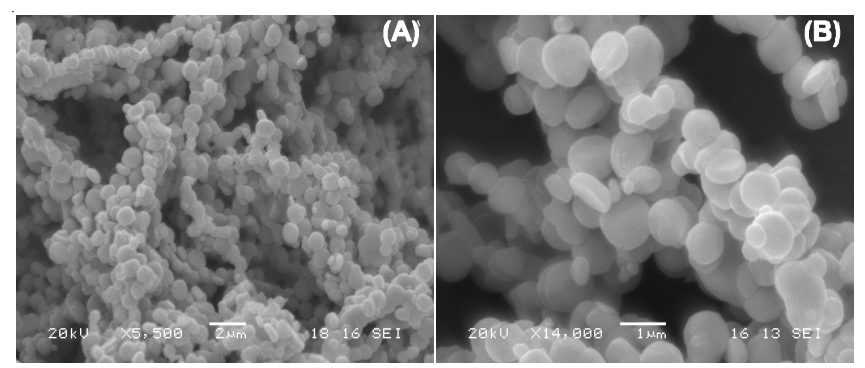

Fig. 2. SEM images of the final samples: (A) The overall morphology of the Ni flakes; (B) The magnified SEM images of the Ni flakes

Magnetic properties of the flake-like Ni products were explored employing a sample-vibrating magnetometer. Magnetization $v s$. applied magnetic field for the flake-like Ni products collected at the end of the reaction was plotted and exhibited in Fig. 3, in which apparent ferromagnetic characteristics at room temperature can be observed. Values of specific saturation magnetization (Ms) and coercivity $(\mathrm{Hc})$ for the Ni flakes were calculated as $21.3 \mathrm{emu} / \mathrm{g}$ and $204.3 \mathrm{Oe}$, respectively. In other word, coercivity of the flake-like Ni samples prepared in the presence of SDBS is higher than that of the bulk Ni sample at room temperature $(\mathrm{Ms}=55 \mathrm{emu} / \mathrm{g}, \mathrm{Hc}=100 \mathrm{Oe})$.

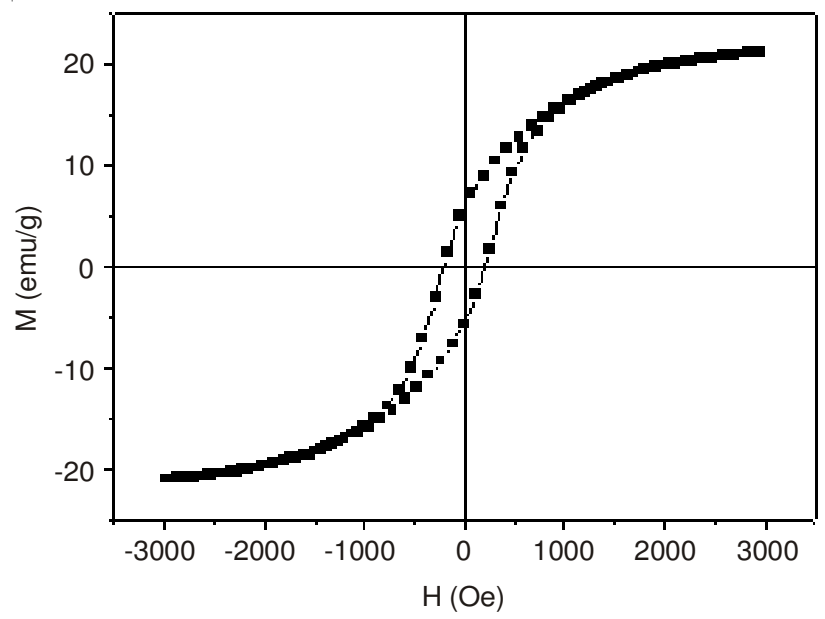

Fig. 3. Hysteresis loop of the final flake-like Ni samples obtained at the end of the reaction measured at room temperature

As is known to all, magnetic properties of the magnetic materials are affected by their crystallite sizes, structures and shapes. In general, Ms of nanoscale magnetic material is lower than that of the bulk material owing to the significantly reducing total magnetic moment originated from the spin disorder on the surface. Meanwhile, the hysteresis loop shape is largely influenced by both the specific surface area of the particles and the magnetic anisotropies including magnetocrystalline, shape, stress, induced and exchange anisotropies. Decreased $\mathrm{Ms}$ of the Ni nanostructures could be well explained as a result. In other word, the obtained representative flake-like $\mathrm{Ni}$ 
assemblies were of apparent shape anisotropy as well as increased coercivity consequently. Thus, the higher coercivity of the flake-like Ni products compared to that of the bulk Ni sample might be ascribed to their unique nanostructures that have been determined by the reduced size and shape anisotropy, which could prevent magnetizing orientally rather than the easily adopted magnetic axes and thus result in a higher coercivity.

\section{Conclusion}

In conclusion, flake-like Ni samples have been prepared by a novel and facile method during a mild reduction procedure in the presence of SDBS. The present method outweighs others in the mild reacting conditions such as normal pressure and declined temperature and thus might be feasible in the synthesis of other magnetic chains. The morphologies and microstructures of the resulting flake-like ultrafine Ni particles have been demonstrated to largely depend on the reaction duration. The flake-like Ni nanostructures were of ferromagnetism and their saturation magnetization (Ms) and coercivity (Hc) values were $21.3 \mathrm{emu} / \mathrm{g}$ and $204.3 \mathrm{Oe}$, respectively, displaying relatively lower saturation magnetization and higher coercivity than those of the bulk Ni sample at room temperature.

\section{REFERENCES}

1. W. Xu, K.Y. Liew, H.F. Liu, T. Huang, C.T. Sun and Y.X. Zhao, Mater. Lett., 62, 2571 (2008).

2. Y. Hu, H.S. Qian, T. Mei, J. Guo and T. White, Mater. Lett., 64, 1095 (2010).

3. T. Hyeon, S.S. Lee, J. Park, Y. Chung and H.B. Na, J. Am. Chem. Soc., 123, 12798 (2001).

4. V.F. Puntes, K.M. Krishnan and A.P. Alivisatos, Science, 291, 2115 (2001).

5. R. Sabirianov, J. Magn. Magn. Mater, 300, 136 (2006).

6. X.M. Lin, H. Claus, U. Welp, I.S. Beloborodov, W.K. Kwok and G.W. Crabtree, J. Phys. Chem. C, 111, 3548 (2007).

7. N. Depalo, P. Carrieri, R. Comparelli, M. Striccoli, A. Agostiano and L. Bertinetti, Langmuir, 27, 6962 (2011).

8. T.J. Zhu, X. Chen, X.Y. Meng, X.B. Zhao and J. He, Cryst. Growth. Des., 10, 3727 (2010).

9. Y. Hou, H. Kondoh, T. Ohta and S. Gao, Appl. Surf. Sci., 241, 218 (2005).

10. G.S. Zhang, X.T. Zhao and L.J. Zhao, Mater. Lett., 66, 267 (2012).

11. R. Yi, R.R. Shi, G.H. Gao, N. Zhang, X.M. Cui and Y.H. He, J. Phys. Chem. C, 113, 1222 (2009).

12. B. Zhang, W. Dai, X.C. Ye, W.Y. Hou and Y.Xie, J. Phys. Chem. B, 109, 22830 (2005)

13. X.M. Ni, Q.B. Zhao, H.G. Zheng, B.B. Li, J.M. Song and D.E. Zhang, Eur. J. Inorg. Chem., 23, 4788 (2005).

14. W. Zhou, L. He, R. Cheng, L. Guo, C.P. Chen and J.L. Wang, J. Phys. Chem. C, 113, 17355 (2009).

15. H.L. Niu, Q.W. Chen, M. Ning, Y.S. Jia and X.J. Wang, J. Phys. Chem. $B, \mathbf{1 0 8}, 3996$ (2004).

16. L.P. Zhu, H.M. Xiao and S.Y. Fu, Eur. J. Inorg. Chem., 25, 3947 (2007). 